\title{
Research Paper: The Effect of 8-Week Strength Training, Balance Training and Combined Training on the Dynamic and Static Balance of the Elderly Inactive Men
}

\author{
${\text { Majid Barzegari }{ }^{1}, \text { Seyed Sadredin Shojaedin }}^{1^{*}} \odot$, Mohammad Bayat Tork ${ }^{2}$ \\ 1. Department of Corrective Exercise and Sport Injuries, School of Physical Education and Sports Sciences, Kharazmi University, Tehran, Iran. \\ 2. Sport Sciences and Physical Education, Faculty of Humanities Science, University of Hormozgan, Bandar Abbas, Iran.
}

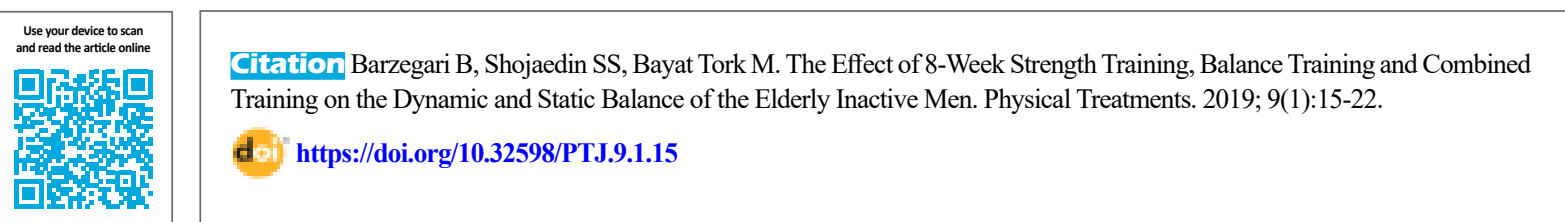

(C) $(0)$

Article info:

Received: 10 May 2018

Accepted: 17 Nov 2018

Available Online: 01 Jan 2019

Keywords:

Strength exercise, Balance exercise, Static balance, Dynamic balance, Elderly

\section{A B S T RA C T}

Purpose: of the most critical changes in the elderly is the weakness in keeping their balance that can expose them to severe injury risks. The purpose of this study was to evaluate the effects of 8 weeks of strength, balance, and combined (strength and balance) training programs on the dynamic and static balance of inactive older men.

Methods: Forty older men, who were the members of Robat Karim Social Security Retirement Association, voluntarily participated in this study. The subjects were randomly assigned to 1 . The strength training group; 2 . The balance training group; 3 . The combined (strength and balance) training group; and 4. The control group. To assess the samples' static balance, we used the Sharpened Romberg test. Also, their dynamic balance was assessed by the Star Excursion Balance Test (SEBT). To analyze the difference in means between groups variables in the pre-test and post-test, the Paired t-test and Analysis of Covariance (ANCOVA) with Tukey's post hoc test were used. The significance level was set at $\mathrm{P} \leq 0.05$.

Results: The results of this study showed that strength, balance, and combined exercise programs improved the dynamic and static balance of older men $(\mathrm{P}=0.001)$.

Conclusion: Balance is essential for everyday activities for all people, especially older adults. To reduce the probability of falling in older adults, we recommend applying strength, balance, and combined exercise programs for improving balance in these people.

* Corresponding Author:

Seyed Sadredin Shojaedin, PhD.

Address: Department of Corrective Exercise and Sport Injuries, School of Physical Education and Sports Sciences, Kharazmi University,Tehran, Iran. Phone: +98 (21) 22228001

E-mail: shojaedin@mu.ac.ir 


\section{Highlights}

- Strength training can improve balance in older adults.

- Balance training can improve balance in older adults.

- Combination of balance and strength training can improve balance in older adults

\section{Plain Language Summary}

The weakness of balance can result in irrecoverable damages, so this study aimed to compare three training programs on the balance of inactive older men. For this purpose, we put the subjects in three different groups, and each of them performed one kind of training program, i.e., strength, balance, and combined (strength and balance) training for 8 weeks. At the end of the protocol, we compared the change in balance in all groups. The result showed that all methods could improve older people's balance significantly, so we recommend these methods for improving balance in older adults.

\section{Introduction}

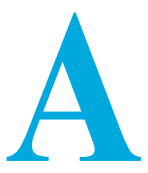

ging is a process with gradual, progressive, and spontaneous loss of most physiological functions of the body [1]. These changes may be due to a decrease in neuromuscular function, muscle mass, strength, endurance, and joint range of movement [2]. Among these impaired functions, we can mention the changes in the systems involved in balance control, which can expose the elderly to severe injuries such as bone fractures and long-term disabilities [3]. Balance is defined as maintaining optimal posture in both static and dynamic positions [4]. Keeping an optimal balance requires a complex interaction between internal factors (proprioception, auditory, and visual senses) and muscular factors. These interactions have a reciprocal effect on the neural network and movement feedbacks [5]. All factors involved in balance are affected by the aging process. Changes in voluntary and involuntary aspects of movement play an important role in this transformation because natural processing and sensory receptors are the most important factors affecting stance and balance control [6].

Previous studies have shown that imbalance in most situations leads to falling, which is one of the most common and serious problems of old age and has physical (pelvic fracture, disability, loss of physical ability, and death), psychological (loss of confidence and self-esteem and decline in life expectancy) and financial consequences [7]. Older adults usually fall several times a year, which may result in fractures, soft tissue injuries, immobility, and eventually, long-term disability and im- mobility, or even death [8]. A review study in 1999 in Iran showed that $19-20 \%$ of older men and $24-36 \%$ of older women had been traumatized, the main causes of which were unwanted incidents and falling [9]. According to the previous studies, the imbalance has been one of the main reasons of falling among the elderly [10], so the researchers have paid attention to the phenomenon of balance among this age group.

Physical activity and exercises are the methods used to prevent, delay, or treat problems caused by the aging process, and their positive impact on the quality of life of older adults has been already documented. Exercise and regular activities in the elderly maintain and improve the function of the cardiovascular system, compensate muscle mass loss and the power (caused by the aging process), provide bone health, improve balance, and increase flexibility [11]. In the meantime, balance exercises, which are mainly done by a balance board, has been introduced as a method for retraining proprioception sense [12]. Also, strength exercises with bolstering ligaments, stabilizing joints, and stimulating the proprioception sense play an essential role in maintaining balance.

An optimal balance is one of the indicators for determining the independence of the older people, so researchers try to investigate and identify the factors affecting balance to increase the independence in movements, promote the safety of everyday and sport physical activities, and prevent damages caused by falling [13]. However, the literature review of studies indicates that various exercises have been tested on the elderly's balance, but different results have gained regarding their effects [14-16]. Besides, there was no study comparing the effects of strength, balance, and 
A

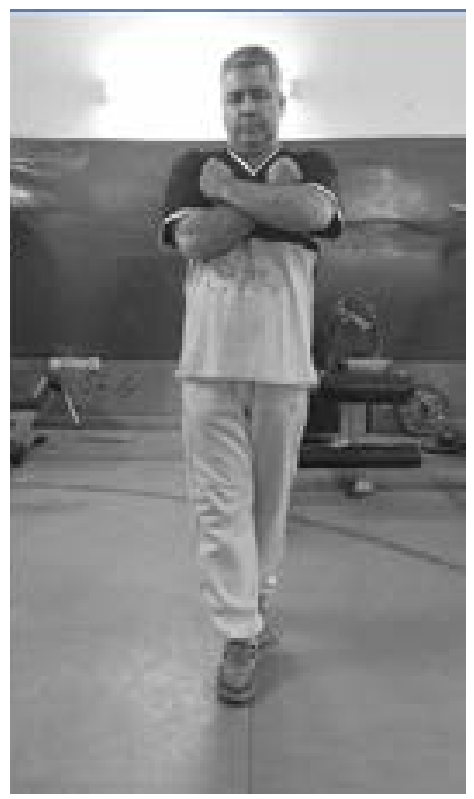

Figure 1. Performing Sharpened Romberg test

A. Closed eyes; B. Open eyes

combination exercises on older adults. Therefore, this study aimed to investigate the efficacy of eight weeks of strength, balance, and combination training on the balance of inactive older adults.

\section{Materials and Methods}

This is a quasi-experimental research with a causalcomparative design using pre-test and post-test. The research model was of an impact assessment type.

\section{Study subjects}

The study population was the retired older men aged 55 to 65 years. Demographic characteristics of subjects presented in Table 2. They were members of the Social Security Pensioners' Association of Robat Karim City, Iran. Of them, 40 subjects were selected as the study sample. They were selected using convenience sampling method and based on health assessment criteria (alertness and functional vestibular tests). Finally, the samples were randomly divided into four groups: 1. Strength training (10 subjects); 2. Balance training (10 subjects); 3. Combination training (10 subjects); and 4. Control group (10 subjects). The participants were asked to provide a complete history of their possible joint dislocation and falling. Considering the purpose of the study, the subjects who were unable to perform cognitive tests (measured by mini-mental state examination), those who had a history of falling over the last 12 months, or suffered from any kind of displacement or joint dislocation, and people who had chronic arthritis or dizziness were excluded from the study $[14,15]$.

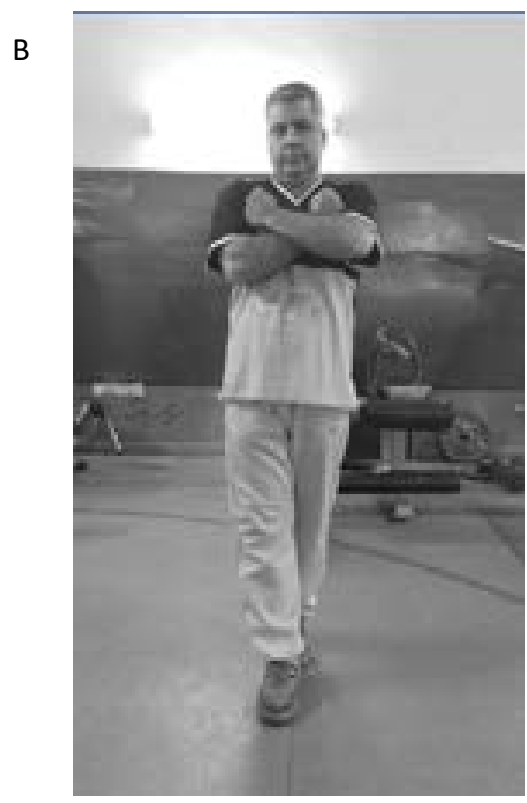

PHYSICAL TREA MENTS

\section{Study procedure}

Before performing any measurements, all participants were asked to sign the consent form for participation in the exercise program. One day before the exercises, the subjects' static balance with open and closed eyes and their dynamic balance were evaluated. The Sharpened Romberg test was used to measure the static balance. It is a test that assesses the integrity of the neural pathways in the spine in neurological conditions. It is frequently used in situations when there is a possibility of disturbance in the proprioception sense. This test easily recognizes a possible pathology in the input pathways of proprioception [17]. The reliability values of the test have been reported to be $0.90-0.91$, and $0.76-0.77$ for eyes open and eyes closed state, respectively (Figure 1) [17, 18]. The method of performing this test is as follows: the subject stands barefoot in such a way that one leg (the dominant leg) is placed ahead of the other leg and the arms are crossed over the chest. The examiner measures how long each subject can hold this position with open or closed eyes and records it as the subject's score [18].

Dynamic balance was measured using get-up and go test. Mathias designed this test in 1986. The scoring scale is 1 to 5 . This test has high reliability for testing the elderly, patients with stroke or Parkinson disease, and screening for falling risk. Score 1 indicates normal performance and score 5 severe disorders [19]. In 1991, the modified version of this test was introduced 


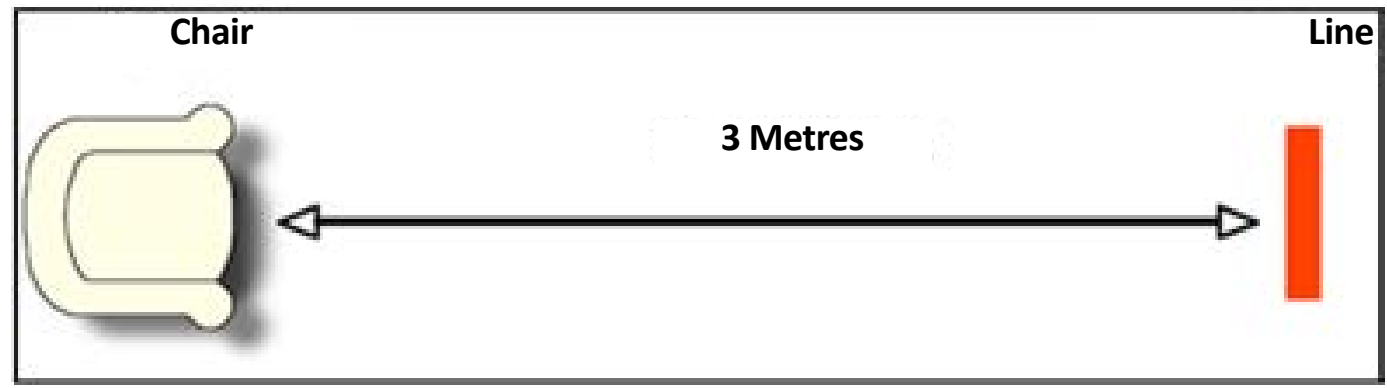

Figure 2. Test run of get-up and go

by Podsialo and Richardson, in which they used a time scale instead of quality scale to calculate the testee score [20]. The intra-rater reliability (0.98-0.99) and inter-rater reliability $(0.97-0.98)$ of the test have been reported, especially for patients, the elderly, and amputee patients $[20,21]$. To implement this test, the patient is required to stand up from an armless chair, without using their hands, and walk $3 \mathrm{~m}$ and then return and sit down on the chair $[20,21]$. The subjects were asked to complete this exercise in the fastest way possible, without running, and then the total test time was recorded. They practiced this test three times each to get acquainted with the test (Figure 2). Then, each subject performed the test three times, and the average score was recorded as his dynamic balance score.

\section{Training programs}

Strength training group performed these exercises 3 days a week for 8 weeks. The exercise session comprised 10 minutes of warm-up (stretching and aerobic exercises) and strength exercises for trunk, thighs and feet extensors as well as trunk, thighs, legs, plantar flexors, and ankle dorsiflexors, adductors and adductors and finally cool down (relaxation). These exercises were adopted from the US National Sports Medicine Program and a consultation with a practitioner. Table 1 shows intensity, repetition, and resting time between movements in different weeks of training. Strength exercise included leg press, bench press, bending leg from the back, stretching the leg, lateral pulldown, seated cable row, and heel lift using gym equipment.

\section{PHYSICAL TREA $\mid$ MENTS}

Balance training group performed exercises in accordance with the method of Seidler (1997) exercises including the following movements in three 30-minute sessions a week [22]:

1. Standing on one leg: standing on one leg while the other leg is placed in line with the mid-foot plantar muscles. If possible, the leg is held in the same position for 30 seconds. This exercise is repeated 3 times on each leg once with open eyes and two times with closed eyes. It is better to do this exercise on the foam rubber.

2. Hyperextension of the neck: Standing while gently turning the neck in the hyperextension mode and then bending it slowly forward. This exercise is repeated 5 times with open eyes, and 5 times with closed eyes and on foam rubber, if possible.

3. Free leg swinging: Standing on one leg while the other leg was moved slightly forward, to the sides, and back. The legs are changed, and this exercise is performed 3 times with open eyes and then 3 times with closed eyes. The subject may first start walking in the form of a glide on the ground; then they were asked to stand entirely on one leg and move the other leg freely.

4. Lifting the heel and toes: movements are performed in a gentle rocking form on toes and holding then back to the heels and hold the position, 3 times repetition with open eyes and 3 times with closed eyes.

Table 1. Intensity, repetition, and resting period of strength exercises

\begin{tabular}{|c|c|c|c|c|}
\hline Week & $\begin{array}{l}\text { Intensity (The Percentage of the Maximum } \\
\text { Strength of one Repetition) (\%) }\end{array}$ & Repetitions & Turns & Resting \\
\hline First and second & $30-40$ & $10-12$ & 2 & \\
\hline Third and fourth & $50-60$ & $8-10$ & 2 & 2-3 minutes after movements \\
\hline Fifth to eighth & $70-80$ & $8-10$ & 2 & \\
\hline
\end{tabular}


Table 2. The demographic characteristics (Mean \pm SD) of subjects specified by groups

\begin{tabular}{|c|c|c|c|c|c|}
\hline \multirow{2}{*}{ Variable } & \multicolumn{4}{|c|}{ Group } & \multirow{2}{*}{$\mathbf{P}$} \\
\hline & Strength Training & Balance Training & Combined Training & Control & \\
\hline Height (cm) & $168.40 \pm 6.04$ & $165.70 \pm 5.73$ & $169.90 \pm 4.90$ & $167.90 \pm 7.35$ & 0.81 \\
\hline Weight (kg) & $69.13 \pm 5.02$ & $69.94 \pm 5.21$ & $71.23 \pm 4.80$ & $69.16 \pm 7.75$ & 0.44 \\
\hline Age $(y)$ & $60.80 \pm 1.67$ & $61.40 \pm 1.95$ & $60.9 \pm 1.96$ & $60.5 \pm 2.27$ & 0.58 \\
\hline
\end{tabular}

5. Gazing at a point: Gazing at a point on the wall at eye level while the neck is turned slowly to the sides, up, and down and also the waist bends to the sides and turns. These exercises are performed 3 times on the foam rubber in standing position.

6. Moving objects: Taking objects from the top while bending back the head, e.g., when something is to be taken from the top shelf, and bending down the head, e.g., when something is to be put on the ground. This exercise is repeated 2 times on the ground and 2 times on the foam.

7. Walking in place: Walking in place on the foam and bending and straightening the head while walking, then 4 times with closed eyes, and each time for 1 minute.

8. Lateral walking: Walking towards the sides, the back leg is almost placed ahead of the front leg.

9. Grapevine Walking: Walking toward the sides, the back leg should be crossed in front of the front leg.

10. Walking backward: Walking backward without look along the back line.

11. Response walking: Walking and then $180^{\circ}$ turn in response to the mark.

Combined training group performed exercises which were a combination of balance and strength exercises for 8 weeks, 3 sessions per week (both strength and balance training in every session) based on both exercise programs. Each training session included 15 minutes of balance exercise and 15 minutes of strength exercise. Two minutes of rest were considered between each type of exercises. Training sessions were under direct observation of two researchers to ensure the correct and accurate performance of the exercises. After completing the 8-week training program, all tests taken from subjects in the pre-test were administered by the third researcher who was blinded to study groups. It should be noted that one subject in the strength training group was removed from the study for not completing the exercises and one from the control group because of not participating in the post-test.

\section{Statistical analysis}

Statistical analysis was done in SPSS 18. After reviewing the normal distribution of data by Shapiro-Wilk test, the covariance analysis test was used to examine the differences between the training groups, and then the Paired t-test was used to examine the within-group changes during pre-test and post-test. The significance level in this study was set at $\leq 0.05$.

\section{Results}

Table 1 presents the demographic characteristics of the subjects, including height, weight, and age in research groups Tables 3 and 4 present the results of the research tests of static balance (with open and closed eyes) and dynamic balance in strength training, balance training, combination training, and control groups. According to the results, static balance (with open and closed eyes) and dynamic balance in all three groups of exercises showed significant differences after applying the specific training protocol for variables each group $(\mathrm{P}=0.001)$. The control group did not show any significant changes between pre-test and post-test.

\section{Discussion}

With increasing age, muscular power is progressively decreasing. Because muscle strength is one of the essential factors in maintaining balance and preventing postural sway, its weakness impairs balance in individuals [23]. The most noticeable fluctuations in automatic postural responses occur in the changes of pelvic angle and fluctuations leading to the taking steps. Since muscle weakness, lack of flexibility, loss of muscle synergy mechanisms and planning, as well as motor control problems, all are responsible for deficiency of balance, 
Table 3. Tukey test results to examine between-group differences for each group

\begin{tabular}{|c|c|c|c|}
\hline Variable & Group & Adjusted Mean \pm SD & Tukey Test Results \\
\hline \multirow{5}{*}{ Static balanc } & Control & $1.02 \pm 0.03$ & $0.56^{\mathrm{a}}$ \\
\hline & Balance training & $0.71 \pm 0.03$ & $0.001^{b}$ \\
\hline & & & \\
\hline & Strength training & $0.78 \pm 0.03$ & $0.001^{c}$ \\
\hline & Combination training & $0.56 \pm 0.06$ & 0.001 \\
\hline \multirow{5}{*}{ Dynamic balar } & Control & $2.79 \pm 0.04$ & $1.00^{\mathrm{k}}$ \\
\hline & Balance training & $2.37 \pm 0.04$ & $0.001^{b}$ \\
\hline & & & \\
\hline & Strength training & $2.35 \pm 0.05$ & $0.001^{c}$ \\
\hline & Combination training & $1.98 \pm 0.09$ & $0.001^{f}$ \\
\hline
\end{tabular}

PHYSICAL TREA $\ M E N T S$

${ }^{\mathrm{a} \& \mathrm{k}}$. Comparing between three groups; ${ }^{\mathrm{b}}$. Comparing between control and balance groups; ${ }^{\mathrm{c}}$. Comparing between control and combination groups; ${ }^{\mathrm{f}}$. Comparing between control and strength groups

applying a physical fitness program is an excellent strategy to prevent the disturbance of the elderly's balance and falling [24]. Rosendall et al. reported that strength and balance training would improve the walking ability, enhance the strength of the lower extremities, and reduce the risk of falling [25].

Also based on the theory of systemic function, control of the body's position in space results from the complex interaction between the nervous system and the musculoskeletal system. In this system, postural control, maintaining balance, and subsequently creating motion requires sensory data to detect the position of the body in space as well as the ability of the musculoskeletal system for applying force. Based on this theory, musculoskeletal factors that affect balance regulation include the range of joint motion, strength, and endurance of the muscle, and biomechanical relationships of various parts [26].
The weakness of each factor leads to a disturbance of static and dynamic balance. Considering the results of previous studies and the current study, the improvement of static and dynamic balance due to strength exercises can be related to the increased strength of the lower limbs, facilitated movement of fast-twitch and large muscles, increased muscle coordination, the imposed exercise stress on neuromuscular systems, and the process of removing spontaneous inhibition, and psychological factors [3, 27]. Improvement of balance in these people can lower the likelihood of falling and inability to do daily work, provide more independence, and increase the quality of life in the elderly.

Moreover, balance exercises helped inactive older adults with static and dynamic balance. It seems that improvement in the static and dynamic balance after performing balance training is related to increased muscle

Table 4. The paired t-test and covariance analysis results for within-group and between-group differences

\begin{tabular}{|c|c|c|c|c|c|c|c|c|c|c|}
\hline Variable & $\begin{array}{l}\text { Control } \\
\text { Group }\end{array}$ & $t(p)$ & $\begin{array}{l}\text { Combination } \\
\text { Training Group }\end{array}$ & $t(p)$ & $\begin{array}{l}\text { Balance Train- } \\
\text { ing Group }\end{array}$ & $t(p)$ & $\begin{array}{c}\text { Strength } \\
\text { Training } \\
\text { Group }\end{array}$ & $t(p)$ & $F(p)$ & $\begin{array}{c}\text { Eta } \\
\text { Squared }\end{array}$ \\
\hline $\begin{array}{c}\text { Static } \\
\text { balance }\end{array}$ & $-0.65(0.49)$ & & $3.99(0.001) *$ & & $2.61(0.001)^{*}$ & & $\begin{array}{c}4.96 \\
(0.001)^{*}\end{array}$ & & $23.4(0.001)^{*}$ & 0.76 \\
\hline Pre-test & $0.24 \pm 1.00$ & & $0.28 \pm 0.98$ & & $0.96 \pm 0.27$ & & & & & \\
\hline Post-test & $1.07 \pm 0.16$ & & $0.18 \pm 0.71$ & & $0.07 \pm 0.77$ & & & & & \\
\hline $95 \% \mathrm{Cl}$ & $1.09,0.95$ & & $0.78,0.64$ & & $0.85,0.70$ & & & & & \\
\hline $\begin{array}{l}\text { Dynamic } \\
\text { balance }\end{array}$ & & $-1.00(0.31)$ & & $9.47(0.001) *$ & & $6.53(0.011)^{*}$ & & & $25.73(0.001)^{*}$ & 0.80 \\
\hline Pre-test & $0.21 \pm 2.70$ & & $0.29 \pm 2.71$ & & $0.37 \pm 2.88$ & & & & $F(p)$ & \\
\hline Post-test & $2.73 \pm 0.23$ & & $0.37 \pm 2.33$ & & $2.46 \pm 0.36$ & & & & $23.4(0.001)^{*}$ & \\
\hline $95 \% \mathrm{Cl}$ & $2.89,2.69$ & & $2.48,2.28$ & & $2.45,2.25$ & & & & & \\
\hline
\end{tabular}


strength, higher range of joints movement, better neural control of movements, and psychological factors and overload on the transmission of information through the central nervous triple sensory systems (visual, vestibular, and somatosensory systems) [3, 27]. According to the findings of this research and the similarity of these results with those of previous studies which reflects the improvement of the static and dynamic balance of the elderly after exercising the strength and balance program, it can be expected that combination exercises are also useful in improving the balance factors of inactive older adults. These types of activities have features of both strength and balance exercises, and as the results revealed, the combination group results were significantly different from the control group.

In general, according to the results of this study and their consistency with the results of previous studies, it can be concluded that all three types of strength, balance, and combination (strength and balance) training can be useful in improving the static and dynamic balance of inactive older adults. Therefore, it is recommended that in designing exercises, all three types of exercise programs be used to improve the older people's balance and to increase the independency of their lives in society. Future studies could investigate the effect of these exercises on inactive older women or, in general, on active older people to generalize the results and recommend these exercises for all elderly groups if the results were positive.

\section{Ethical Considerations}

\section{Compliance with ethical guidelines}

This study was confirmed by ethic committee of Kharazmi University.

\section{Funding}

This research did not receive any specific grant from funding agencies in the public, commercial, or not-forprofit sectors.

\section{Authors' contributions}

Conceptualization, methodology, and investigation: All authors; Writing-original draft: Majid Barzegari; Writing-review \& editing: Seyed Sadredin Shojaedin and Mohammad Bayat Tork; Funding acquisition and resources: All authors; Supervision: Seyed Sadredin Shojaedin.

\section{Conflict of interests}

The authors declared no conflict of interest.

\section{Acknowledgments}

Authors appreciate Robat Karim Social Security Retirement Association authorities for their cooperation in conducting this study.

\section{References}

[1] Stevens JA, Olson S. Reducing falls and resulting hip fractures among older women. Home Care Provider. 2000; 5(4):134-41. [DOI:10.1067/mhc.2000.109232]

[2] de Souza Vale RG, de Oliveira RD, Pernambuco CS, da Silva Novaes J, de Andrade AD. Effects of muscle strength and aerobic training on basal serum levels of IGF-1 and cortisol in elderly women. Archives of Gerontology and Geriatrics. 2009; 49(3):343-7. [DOI:10.1016/j.archger.2008.11.011] [PMID]

[3] Shumway-Cook A, Woollacott MH. Theory and practical applications. Philadelphia: Lippincott Williams \& Wilkins; 2001.

[4] Nagy E, Feher-Kiss A, Barnai M, Domján-Preszner A, Angyan L, Horvath G. Postural control in elderly subjects participating in balance training. European Journal of Applied Physiology. 2007; 100(1):97-104. [DOI:10.1007/s00421-0070407-x] [PMID]

[5] Khoda MF, Alijani E, Kohandel M. The effect of reflexology training on postural stability and postural sway in elderly women. European Journal of Experimental Biology. 2014; 4(1):198-202.

[6] Benjuya N, Melzer I, Kaplanski J. Aging-induced shifts from a reliance on sensory input to muscle cocontraction during balanced standing. The Journals of Gerontology Series A: Biological Sciences and Medical Sciences. 2004; 59(2):M16671. [DOI:10.1093/gerona/59.2.M166] [PMID]

[7] Resende SM, Rassi CM. Effects of hydrotherapy in balance and prevention of falls among elderly women. Brazilian Journal of Physical Therapy. 2008; 12(1):57-63. [DOI:10.1590/ S1413-35552008000100011]

[8] Schoenfelder DP, Rubenstein LM. An exercise program to improve fall-related outcomes in elderly nursing home residents. Applied Nursing Research. 2004; 17(1):21-31. [DOI:10.1016/j.apnr.2003.10.008] [PMID]

[9] Reid KF, Fielding RA. Skeletal muscle power: A critical determinant of physical functioning in older adults. Exercise and Sport Sciences Reviews. 2012; 40(1):4-12. [DOI:10.1097/ JES.0b013e31823b5f13] [PMID] [PMCID]

[10] Hobeika CP. Equilibrium and balance in the elderly. ENT: Ear, Nose \& Throat Journal. 1999; 78(8):557-64. [DOI:10.1177 /014556139907800810] 
[11] Stewart KJ. Physical activity and aging. Annals of the New York Academy of Sciences. 2005; 1055(1):193-206. [DOI:10.1196/annals.1323.029] [PMID]

[12] Laskowski ER, Newcomer-Aney K, Smith J. Refining rehabilitation with proprioception training: Expediting return to play. The Physician and Sportsmedicine. 1997; 25(10):89-102. [DOI:10.3810/psm.1997.10.1476] [PMID]

[13] Matsumura BA, Ambrose AF. Balance in the elderly. Clinics in Geriatric Medicine. 2006; 22(2):395-412. [DOI:10.1016/j. cger.2005.12.007] [PMID]

[14] Contant B. The effectiveness of two types of balance-boards to improve elderly balance over an 8-week training intervention [PhD. dissertation]. Ottawa, Canada: University of Ottawa; 2006

[15] Myer GD, Ford KR, Brent JL, Hewett TE. The effects of plyometric vs. dynamic stabilization and balance training on power, balance, and landing force in female athletes. Journal of Strength and Conditioning Research. 2006; 20(2):345-53. [DOI:10.1519/R-17955.1] [PMID]

[16] de Bruin ED, Murer K. Effect of additional functional exercises on balance in elderly people. Clinical Rehabilitation. 2007; 21(2):112-21. [DOI:10.1177/0269215506070144] [PMID]

[17] Clark S, Rose DJ, Fujimoto K. Generalizability of the limits of stability test in the evaluation of dynamic balance among older adults. Archives of Physical Medicine and Rehabilitation. 1997; 78(10):1078-84. [DOI:10.1016/S00039993(97)90131-3]

[18] Steffen T, Seney M. Test-retest reliability and minimal detectable change on balance and ambulation tests, the 36-item short-form health survey, and the unified Parkinson disease rating scale in people with parkinsonism. Physical Therapy. 2008; 88(6):733-46. [DOI:10.2522/ptj.20070214] [PMID]

[19] Nordin E, Lindelöf N, Rosendahl E, Jensen J, Lundin-Olsson L. Prognostic validity of the Timed Up-and-Go test, a modified Get-Up-and-Go test, staff's global judgement and fall history in evaluating fall risk in residential care facilities. Age and ageing. 2008; 37(4):442-8. [DOI:10.1093/ageing/ afn101] [PMID]

[20] Podsiadlo D. The timed "Up \& Go": A test of basic functional mobility for frail elderly persons. Journal of the American Geriatrics Society. 1991; 39(2):142-8. [DOI:10.1111/j.1532-5415.1991.tb01616.x] [PMID]

[21] Langley FA, Mackintosh SF. Functional balance assessment of older community dwelling adults: A systematic review of the literature. Internet Journal of Allied Health Sciences and Practice. 2007; 5(4):1-11.

[22] Seidler RD, Martin PE. The effects of short term balance training on the postural control of older adults. Gait \& Posture. 1997; 6(3):224-36. [DOI:10.1016/S0966-6362(97)00012$\mathrm{X}]$

[23] Lee DK, Kim GM, Ha SM, Oh JS. Correlation of the Y-balance test with lower-limb strength of adult women. Journal of Physical Therapy Science. 2014; 26(5):641-3. [DOI:10.1589/ jpts.26.641] [PMID] [PMCID]

[24] Melzer I, Benjuya N, Kaplanski J. Postural stability in the elderly: A comparison between fallers and non-fallers. Age and Ageing. 2004; 33(6):602-7. [DOI:10.1093/ageing/afh218] [PMID]
[25] Rosendahl E. Fall prediction and a high-intensity functional exercise programme to improve physical functions and to prevent falls among older people living in residential care facilities [PhD. dissertation]. Umeå, Sweden: Umeå University; 2006.

[26] Shumway-Cook A, Gruber W, Baldwin M, Liao S. The effect of multidimensional exercises on balance, mobility, and fall risk in community-dwelling older adults. Physical Therapy. 1997; 77(1):46-57. [DOI:10.1093/ptj/77.1.46] [PMID]

[27] de Rossiter Corrêa MB, Jacobina CB, Da Silva ER, Lima AM. Vector control strategies for single-phase induction motor drive systems. IEEE Transactions on Industrial Electronics. 2004; 51(5):1073-80. [DOI:10.1109/TIE.2004.834973] 